\title{
Pressure Transient Analysis of Multi-Fractured Horizontal Well in Tight Gas Reservoirs
}

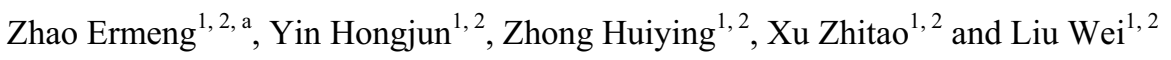 \\ ${ }^{1}$ Key Laboratory of Ministry of Education PRC, Northeast Petroleum University, Daqing, Heilongjiang 163318, China \\ ${ }^{2}$ Accumulation and Development of Unconventional Oil and Gas, State Key Laboratory Cultivation Base Jointly-constructed by \\ Heilongjiang Province and the Ministry of Science and Technology, Daqing, Heilongjiang 163318, China
}

\begin{abstract}
Multi-fractured horizontal well is applied in tight gas reservoirs due to the low permeability. A new pressure transient model of multi-fractured horizontal well based on discrete-fracture model in which the hydraulic fractures are discretized as $2 \mathrm{D}$ entities is built in this paper, The model is divided into hydraulic fracture region and formation region. The model can be solved using the Galerkin finite element method, then the pressure transient type curves are plotted by computer programming. The results show that there are five different flow regimes observed in type curves including early linear flow, early radial flow, elliptical flow, later pseudo-radial flow and boundary response regime. A sensitivity analysis is conducted to study impacts of hydraulic fracture number, hydraulic fracture half-length, hydraulic fracture spacing, and hydraulic fracture conductivity on pressure transient type curves. The new model and obtained results in this paper not only enrich the well testing models, but also play a guiding role in analyzing pressure transient response of multi-fractured horizontal well in tight gas reservoirs.
\end{abstract}

\section{Introduction}

The research on tight gas reservoirs becomes the hot spot in petroleum development. Tight gas reservoirs have low matrix permeability, horizontal wells with multiple hydraulic fracturing technology have been an effect way to produce from tight gas reservoirs.

The pressure transient behavior of multi-fractured horizontal well has been investigated a lot, and several flow models have been developed over the past decades. Gringarten firstly solved the flow problems of two and three dimensions with Green's functions with Green's functions ${ }^{[1]}$. Ozkan developed a new procedure to generate the solutions of flow equations in porous media combining Laplace transformation with source function $^{[2]}$. Based on Gringarten 's approach, Raghavan presented a semi-analytical model to study the pressure transient response characteristic of multi-fractured horizontal wells ${ }^{[3]}$. Zerzar combined the Laplace transformation and source function to present an analytical model for fractured horizontal well, the reservoir can be anisotropic or homogenous or naturally fractured, boundary can be closed or semi-infinite ${ }^{[4]}$. AlKobaisi presented a numerical-analytical flow model of multi-fractured horizontal wells intercepted by a vertical fracture $^{[5]}$. Amini developed a distributed volumetric sources method to solve the flow problems of horizontal wells with multi-stage fractures in a box-shaped reservoir ${ }^{[6]}$. Zhao used source function to analyze the transient pressure response of the multi-fractured horizontal well in shale gas reservoir ${ }^{[7]}$. Yao and Liu obtained the analytical solution of pressure in a horizontal well with multiple hydraulic fractures by using mirror-injection and superposition theorem ${ }^{[8]}$.

Ozkan and Brown developed a tri-linear flow model to study multi-fractured horizontal wells performance ${ }^{[9-}$ ${ }^{10]}$. Stalgorova and Mattar improved the tri-linear flow model to simulate fractures that is surrounded by a simulated region of limited extendibility ${ }^{[1]}$. Later, Stalgorova and Mattar extended the tri-linear flow model to a five regions flow model ${ }^{[12]}$. Then some researchers (Samandarli et al, 2014; Clarkson et al, 2013, 2014) successfully used these models to study the complex well behavior exhibited in the field ${ }^{[13-15]}$. Recently, Sureshjani and Clarkson developed a new enhanced fracture-region model based on the five regions flow model, and the new model is more general and rigorous than previous models ${ }^{[16]}$. These models are simple and flexible, however, the models assume that all fractures have the same properties (fracture half-length, conductivity, and fracture aperture, etc.), as a result, some flow regimes cannot be observed.

In this paper, we present a new flow model based on discrete-fracture method of multi-fractured horizontal well in tight gas reservoirs, and the Galerkin finite element method is applied to solve the model. This model can accurately and effectively describe the effects of transient pressure behavior of multi-fractured horizontal wells, which can provide theoretical guidance for the development of multi-fractured horizontal well in tight gas reservoirs.

\footnotetext{
a Corresponding author: Zhao Ermeng18745953495@163.com
} 


\section{Theoretical model}

\subsection{Physical model}

Fig. 1 illustrates the physical model of multifractured horizontal well used as the basis for the mathematical model developed in this work. The length of horizontal well is $L$, and the net pay thickness is $h$. The gas reservoir is stimulated with $n_{\mathrm{f}}$ hydraulic fractures, hydraulic fracture half length is $x_{\mathrm{f}}$, and the space between fractures is $d_{\mathrm{f}}$.

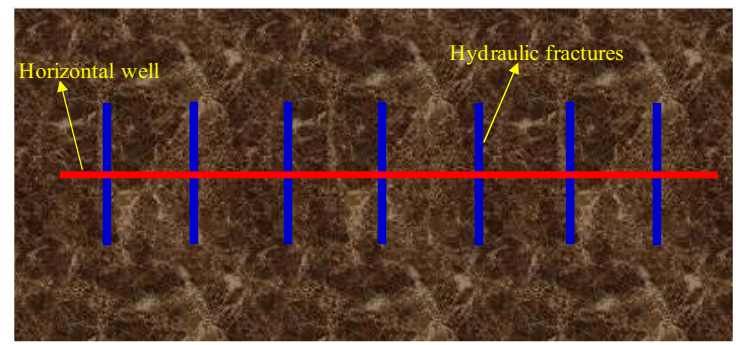

Figure 1. Physical model diagram of multi-fractured horizontal well in tight gas reservoir

The following assumptions are made to simplify the derivation of mathematical model: (1) The reservoir is homogeneous, isotropic, and bounded by an upper and lower closed boundary;(2) The reservoir is composite with the hydraulic fracture region and formation region; (3)The reservoir is produced through a multi-fractured horizontal well, and the wellbore is intersected by several fully penetrating vertical fractures; (4) The gas flow follows Darcy's law and the gravitational and frictional effects is neglected.

\subsection{Mathematical model}

The mass conservation equation is given by

$$
-\nabla \cdot(\rho v)-\rho q \delta\left(M-M^{\prime}\right)=\frac{\partial(\rho \phi)}{\partial t}
$$

where, $\nabla \cdot$ is the divergence operator, $\rho q$ is a source term, $\rho$ is the gas density, $q$ is the flow rate per unit volume, $\delta$ is the Dirac delta function, if $M$ equal $M^{\prime}$, $\delta\left(M-M^{\prime}\right)$ is 1 , otherwise $\delta\left(M-M^{\prime}\right)$ is 0 .

The Darcy's Law in cartesian coordinate is given by

$$
v=-\frac{K}{\mu} \nabla p
$$

The rock compressibility is defined by

$$
\phi=\frac{1}{\phi} \frac{\mathrm{d} \phi}{\mathrm{d} p}
$$

After integration, it is given by

$$
\phi=\phi_{\mathrm{i}} \mathrm{e}^{C_{\mathrm{f}}\left(p-p_{\mathrm{i}}\right)}
$$

The real gas density can be written as

$$
\rho=\frac{p M}{Z R T}
$$

Where $M$ is the molecular weight of gas, $R$ is the gas constant, $Z$ is the gas deviation factor, $T$ is the temperature.

The isothermal gas compressibility can be expressed as follows

$$
C_{\mathrm{g}}=\frac{1}{\rho} \frac{\mathrm{d} \rho}{\mathrm{d} p}=\frac{1}{p}-\frac{1}{Z}\left(\frac{\mathrm{d} Z}{\mathrm{~d} p}\right)_{T}
$$
that

Substituting (2), (4), (5) and (2) into (1), we can see

$$
\nabla \cdot\left(K \frac{p}{\mu Z} \nabla \cdot p\right)-\frac{p}{Z} q \delta\left(M-M^{\prime}\right)=\frac{p}{Z} \phi C_{\mathrm{t}} \frac{\partial p}{\partial t}
$$

Where, $C_{\mathrm{t}}$ is the total compressibility, $C_{\mathrm{t}}=C_{\mathrm{g}}+C_{\mathrm{f}}$.

To simply the equation, the real gas pseudo-pressure is introduced, which can be defined as

$$
\psi(p)=2 \int_{p_{0}}^{p} \frac{p}{\mu Z} \mathrm{~d} p
$$

The diffusivity equation becomes

$$
K \Delta \psi-\frac{2 p}{Z} q \delta\left(M-M^{\prime}\right)=\phi \mu C_{\mathrm{t}} \frac{\partial \psi}{\partial t}
$$

Where, $\Delta$ is the Laplacian operator, $\Delta=\frac{\partial^{2}}{\partial x^{2}}+\frac{\partial^{2}}{\partial y^{2}}+\frac{\partial^{2}}{\partial z^{2}}$.

For hydraulic fractures, the diffusivity equation of pseudo-pressure can be written as

$$
K_{\mathrm{F}} \Delta \psi_{\mathrm{F}}-\frac{2 p}{Z} q \delta\left(M-M^{\prime}\right)=\phi_{\mathrm{F}} \mu C_{\mathrm{tF}} \frac{\partial \psi_{\mathrm{F}}}{\partial t}
$$

The initial condition for this problem is

$$
\left.\psi_{\mathrm{F}}\right|_{t=0}=\psi_{\mathrm{i}}
$$

Where, $\psi_{\mathrm{i}}$ is the original reservoir pseudo-pressure.

It is assumed that the hydraulic fractures and reservoir region are perfectly connected, so that pseudopressure is continuous at the interfaces

$$
\psi_{\mathrm{F}}\left|\partial \Omega_{\mathrm{R}} \mathrm{I} \partial \Omega_{\mathrm{F}}=\psi_{\mathrm{R}}\right| \partial \Omega_{\mathrm{R}} \mathrm{I} \partial \Omega_{\mathrm{F}}
$$
by

The diffusivity equation for reservoir region is given

$$
K_{\mathrm{R}} \Delta \psi_{\mathrm{R}}=\phi_{\mathrm{R}} \mu C_{\mathrm{tR}} \frac{\partial \psi_{\mathrm{R}}}{\partial t}
$$

The initial condition is given by

$$
\left.\psi_{\mathrm{f}}\right|_{t=0}=\left.\psi_{\mathrm{m}}\right|_{t=0}=\psi_{\mathrm{i}}
$$

Pseudo-pressure continuous condition between the natural fractures and hydraulic fractures is given by 


$$
\left.\psi_{\mathrm{f}}\right|_{\partial \Omega_{\mathrm{f}} \mathrm{I} \partial \Omega_{\mathrm{F}}}=\left.\psi_{\mathrm{F}}\right|_{\partial \Omega_{\mathrm{f}} \mathrm{I} \partial \Omega_{\mathrm{F}}}
$$

No-flow condition at the outer reservoir boundary is given by

$$
\left.\frac{\partial \psi_{\mathrm{R}}}{\partial x}\right|_{x=x_{\mathrm{e}}}=\left.\frac{\partial \psi_{\mathrm{R}}}{\partial y}\right|_{y=y_{\mathrm{e}}}=\left.\frac{\partial \psi_{\mathrm{R}}}{\partial z}\right|_{z=z_{\mathrm{e}}}=0
$$

Dimensionless variables are defined by

$$
\begin{aligned}
& \psi_{j \mathrm{D}}=\frac{\pi K_{\mathrm{f}} h T_{\mathrm{sc}}}{q_{\mathrm{sc}} p_{\mathrm{sc}} T}\left(\psi_{\mathrm{i}}-\psi_{j}\right) \\
& t_{\mathrm{D}}=\frac{K_{\mathrm{R}} t}{\phi_{\mathrm{R}} \mu C_{\mathrm{tR}} L^{2}}, x_{\mathrm{D}}=\frac{x}{L}, \\
& y_{\mathrm{D}}=\frac{y}{L}, z_{\mathrm{D}}=\frac{z}{L}, q_{\mathrm{D}}=\frac{q Z_{\mathrm{sc}} T_{\mathrm{sc}} p_{\mathrm{wf}}}{q_{\mathrm{sc}} Z T p_{\mathrm{sc}}} h L^{2} .
\end{aligned}
$$

Where, F, R are hydraulic fractures and reservoir region respectively.

So the dimensionless mathematical model of hydraulic fractures can be written as

$$
\left\{\begin{array}{l}
\frac{K_{\mathrm{F}}}{K_{\mathrm{R}}} \Delta \psi_{\mathrm{FD}}+2 \pi q_{\mathrm{D}} \delta\left(M_{\mathrm{D}}-M_{\mathrm{D}}^{\prime}\right)=\frac{\phi_{\mathrm{F}} C_{\mathrm{tF}}}{\phi_{\mathrm{R}} C_{\mathrm{tR}}} \frac{\partial \psi_{\mathrm{FD}}}{\partial t_{\mathrm{D}}} \\
\left.\psi_{\mathrm{FD}}\right|_{t_{\mathrm{D}}=0}=0 \\
\left.\psi_{\mathrm{FD}}\right|_{\partial \Omega_{\mathrm{R}} \mathrm{I} \partial \Omega_{\mathrm{F}}}=\left.\psi_{\mathrm{RD}}\right|_{\partial \Omega_{\mathrm{R}} \mathrm{I} \partial \Omega_{\mathrm{F}}}
\end{array}\right.
$$

Dimensionless mathematical model of reservoir region can be written as

$$
\left\{\begin{array}{l}
\Delta \psi_{\mathrm{RD}}=\frac{\partial \psi_{\mathrm{RD}}}{\partial t_{\mathrm{D}}} \\
\left.\psi_{\mathrm{RD}}\right|_{t_{\mathrm{D}}=0}=0 \\
\left.\psi_{\mathrm{RD}}\right|_{\partial \Omega_{\mathrm{R}} \mathrm{I} \partial \Omega_{\mathrm{F}}}=\left.\psi_{\mathrm{FD}}\right|_{\partial \Omega_{\mathrm{R}} \mathrm{I} \partial \Omega_{\mathrm{F}}} \\
\left.\frac{\partial \psi_{\mathrm{RD}}}{\partial x_{\mathrm{D}}}\right|_{x_{\mathrm{D}}=x_{\mathrm{eD}}}=\left.\frac{\partial \psi_{\mathrm{RD}}}{\partial y_{\mathrm{D}}}\right|_{y_{\mathrm{D}}=y_{\mathrm{eD}}}=\left.\frac{\partial \psi_{\mathrm{RD}}}{\partial z_{\mathrm{D}}}\right|_{z_{\mathrm{D}}=z_{\mathrm{eD}}}=0
\end{array}\right.
$$

\section{Solution of the mathematical model}

In this paper, hydraulic fractures are described by discrete fracture model (DFM). The main idea of the DFM is a $2 \mathrm{D}$ representation of the fractures ${ }^{[17]}$.

Galerkin weighted residual method and finiteelement discretization are used in this work to solve the equations. The reservoir geometry is discretized using tetrahedron elements for the reservoir region and triangular elements for the hydraulic fractures.

The hydraulic fracture element pseudo-pressure is approximated as

$$
\psi_{\mathrm{FD}}^{e}=N_{\mathrm{F} 1} \psi_{\mathrm{FD} 1}^{e}+N_{\mathrm{F} 2} \psi_{\mathrm{FD} 2}^{e}+N_{\mathrm{F} 3} \psi_{\mathrm{FD} 3}^{e}
$$

Where $\psi_{\mathrm{FD} 1}^{e}, \psi_{\mathrm{FD} 2}^{e}$, and $\psi_{\mathrm{FD} 3}^{e}$ are pseudo-pressure of the three nodes, $N_{\mathrm{F} 1}, N_{\mathrm{F} 2}$, and $N_{\mathrm{F} 3}$ are piecewise-linear interpolation functions defined on triangles.
Then, the discrete form of the Eq. 17 can be written as

$$
\begin{aligned}
& w \frac{K_{\mathrm{F}}}{K_{\mathrm{f}}} \int_{\Omega_{F, e}} \nabla \boldsymbol{N}_{e, F}^{\mathrm{T}} \nabla \boldsymbol{N}_{e, F} \mathrm{~d} \bar{\Omega}_{F, e} \boldsymbol{\psi}_{\mathrm{FD}}^{e} \\
& +w \frac{\phi_{\mathrm{F}} C_{\mathrm{tF}}}{\phi_{\mathrm{m}} C_{\mathrm{tm}}+\phi_{\mathrm{f}} C_{\mathrm{tf}}} \iint_{\bar{\Omega}_{F, e}} \boldsymbol{N}_{e, F}^{\mathrm{T}} \boldsymbol{N}_{e, F} \mathrm{~d} \bar{\Omega}_{F, e} \frac{\partial \boldsymbol{\psi}_{\mathrm{FD}}^{e}}{\partial t_{\mathrm{D}}} \\
& -2 \pi w \iint_{\Omega_{F, e}} N_{e, F}^{\mathrm{T}} q_{\mathrm{FD}} \delta\left(M_{\mathrm{D}}-M_{\mathrm{D}}^{\prime}\right) \mathrm{d} \bar{\Omega}_{F, e}=0
\end{aligned}
$$

Where $\boldsymbol{N}_{e, F}=\left[\begin{array}{lll}N_{\mathrm{F} 1} & N_{\mathrm{F} 2} & N_{\mathrm{F} 3}\end{array}\right], \boldsymbol{\psi}_{\mathrm{FD}}^{e}=\left[\begin{array}{lll}\psi_{\mathrm{FD} 1}^{e} & \psi_{\mathrm{FD} 2}^{e} & \psi_{\mathrm{FD} 3}^{e}\end{array}\right]^{\mathrm{T}}$,

The same operation is implemented to tetrahedron elements for reservoir region. The pseudo-pressure of formation region is approximated as

$$
\psi_{\mathrm{RD}}^{e}=N_{\mathrm{R} 1} \psi_{\mathrm{RD} 1}^{e}+N_{\mathrm{R} 2} \psi_{\mathrm{RD} 2}^{e}+N_{\mathrm{R} 3} \psi_{\mathrm{RD} 3}^{e}+N_{\mathrm{R} 4} \psi_{\mathrm{RD} 4}^{e}
$$

Where $\psi_{\mathrm{RD} 1}^{e}, \psi_{\mathrm{RD} 2}^{e}, \psi_{\mathrm{RD} 3}^{e}$, and $\psi_{\mathrm{RD} 4}^{e}$ are four pseudopressure of reservoir region, $N_{\mathrm{R} 1}, N_{\mathrm{R} 2}, N_{\mathrm{R} 3}$, and $N_{\mathrm{R} 4}$ are piecewise-linear interpolation functions defined on tetrahedrons.

Then, the discrete form of Eq.18 are as follows

$$
\iiint_{\Omega_{R, e}} \nabla \boldsymbol{N}_{e, R}^{\mathrm{T}} \nabla \boldsymbol{N}_{e, R} \mathrm{~d} \Omega_{R, e} \boldsymbol{\psi}_{\mathrm{RD}}^{e}+\iiint_{\Omega_{R, e}} \boldsymbol{N}_{e, R}^{\mathrm{T}} \boldsymbol{N}_{e, R} \mathrm{~d} \Omega_{R, e} \frac{\partial \boldsymbol{\psi}_{e, \mathrm{RD}}}{\partial t_{\mathrm{D}}}=0
$$

Where, $N_{e, R}=\left[N_{\mathrm{R} 1} N_{\mathrm{R} 2} N_{\mathrm{R} 3} N_{\mathrm{R} 4}\right]$,

$$
\boldsymbol{\psi}_{\mathrm{RD}}^{e}=\left[\begin{array}{llll}
\psi_{\mathrm{RD} 1}^{e} & \psi_{\mathrm{RD} 2}^{e} & \psi_{\mathrm{RD} 3}^{e} & \psi_{\mathrm{RD} 4}^{e}
\end{array}\right]^{\mathrm{T}} \text {, }
$$

Combining the equations of reservoir region and hydraulic fractures by the relationship of nodes, the only thing we should notice is that hydraulic fractures and reservoir region have the same node, as below

$$
\boldsymbol{A}^{R} \boldsymbol{\psi}_{\mathrm{RD}}+\boldsymbol{B}^{R} \frac{\partial \boldsymbol{\psi}_{\mathrm{RD}}}{\partial t_{\mathrm{D}}}=\boldsymbol{Q}^{f}
$$

Where,

$$
\begin{aligned}
& \boldsymbol{A}^{R}=\sum_{e=1}^{N_{R}} \iiint_{\Omega_{R, e}} \nabla \boldsymbol{N}_{e, R}^{\mathrm{T}} \nabla \boldsymbol{N}_{e, R} \mathrm{~d} \Omega_{R, e}+w \frac{K_{\mathrm{F}}}{K_{\mathrm{R}}} \sum_{e=1}^{N_{F}} \iint_{\bar{\Omega}_{F, e}} \nabla \boldsymbol{N}_{e, F}^{\mathrm{T}} \nabla \boldsymbol{N}_{e, F} \mathrm{~d} \bar{\Omega}_{F, e}, \\
& \boldsymbol{B}^{R}=\sum_{e=1}^{N_{R}} \iiint_{\Omega_{R, e}} N_{e, R}^{\mathrm{T}} \boldsymbol{N}_{e, R} \mathrm{~d} \Omega_{R, e}+\frac{w \phi_{\mathrm{F}} C_{\mathrm{tF}}}{\phi_{\mathrm{R}} C_{\mathrm{tR}}} \sum_{e=1}^{N_{F}} \iint_{\bar{\Omega}_{F, e}} N_{e, F}^{\mathrm{T}} \boldsymbol{N}_{e, F} \mathrm{~d} \bar{\Omega}_{F, e}, \\
& \boldsymbol{Q}^{R}=2 \pi w \sum_{e=1}^{N_{F}} \iint_{\Omega_{F, e}} \boldsymbol{N}_{e, F}^{\mathrm{T}} q_{\mathrm{FD}} \delta\left(M_{\mathrm{D}}-M_{\mathrm{D}}^{\prime}\right) \mathrm{d} \bar{\Omega}_{F, e} .
\end{aligned}
$$

To get the solutions, the time derivative terms are discretized by forward difference. We can obtain the $(n+1)$ th pseudo-pressure of reservoir region by nth pseudo-pressure, as below

$$
\left(\boldsymbol{A}^{R}+\frac{\boldsymbol{B}^{R}}{\Delta t_{n}}\right) \boldsymbol{\psi}_{\mathrm{RD}}^{n+1}=\boldsymbol{Q}^{R}+\frac{\boldsymbol{B}^{R}}{\Delta t_{n}} \boldsymbol{\psi}_{\mathrm{RD}}^{n}
$$

Where $\Delta t_{\mathrm{n}}$ is the timestep. 


\section{Discussion of the results}

As shown in Fig 2, the pseudo-pressure and its derivative curves can be divided into five flow regimes. (1) Early linear flow regime, the pseudo-pressure and its derivative curve have a slope of 1/2;(2) Early radial flow regime, the pseudo-pressure derivative curve is seems like a horizontal straight line;(3) Elliptical flow regime, which is characterized by a slope of 0.36 on the pseudopressure derivative curve in this regime;(4) Later pseudo-radial flow regime, the pseudo-pressure derivative curve is almost a horizontal straight line;(5) Boundary response regime, the pressure has spread to the outer boundary for closed outer boundary, the pseudo-pressure and its derivative curve tend to an asymptote with a slope of 1 .

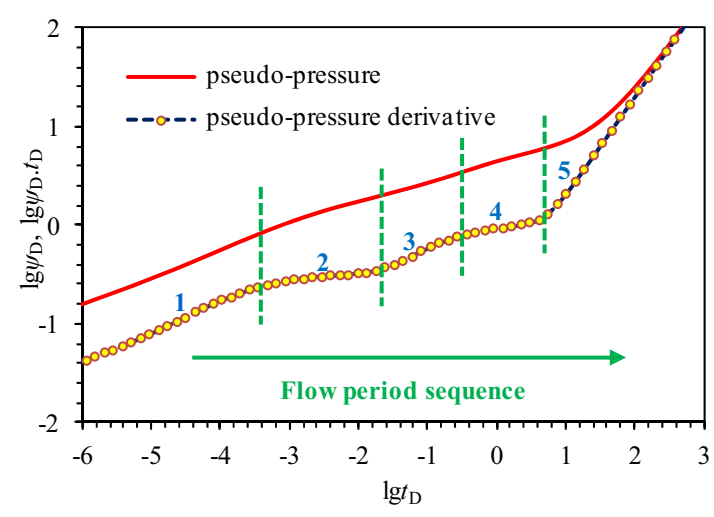

Figure 2. Pseudo-pressure and its derivative curves

Fig. 3 shows the effect of hydraulic fracture number $\left(n_{\mathrm{f}}\right)$ on pseudo-pressure and its derivative curves. As shown in Fig.3, the $n_{\mathrm{f}}$ has great effect on early and intermediate regimes of the curves, with the decrease of $n_{\mathrm{f}}$, the occurrence time of elliptical flow shows later, even disappears when there is only one hydraulic fracture. Meanwhile, pressure drop will be smaller for more hydraulic fractures because the increased formation permeability around wellbore caused by hydraulic fractures will decrease the pressure drop around wellbore.

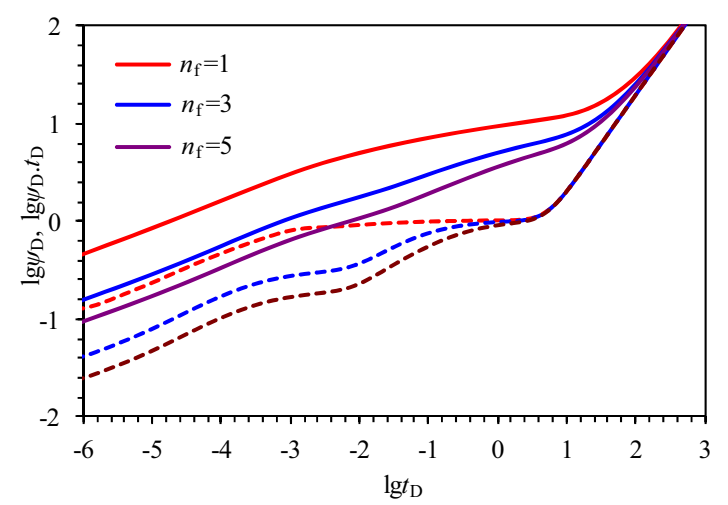

Figure 3. Effect of $n_{\mathrm{f}}$ on the type curves

Fig.4 shows the effect of hydraulic fracture halflength $\left(x_{\mathrm{f}}\right)$ on pseudo-pressure and its derivative curves. The effect of $x_{\mathrm{f}}$ on the curves is mainly on early linear flow and early radial flow regime, with the decrease of $x_{\mathrm{f}}$, the appearance time of early radial flow will be earlier, and the longer the duration will last. In addition, the pressure drop will become larger for a bigger $x_{\mathrm{f}}$, because the formation permeability will be bigger around wellbore caused by hydraulic fractures.

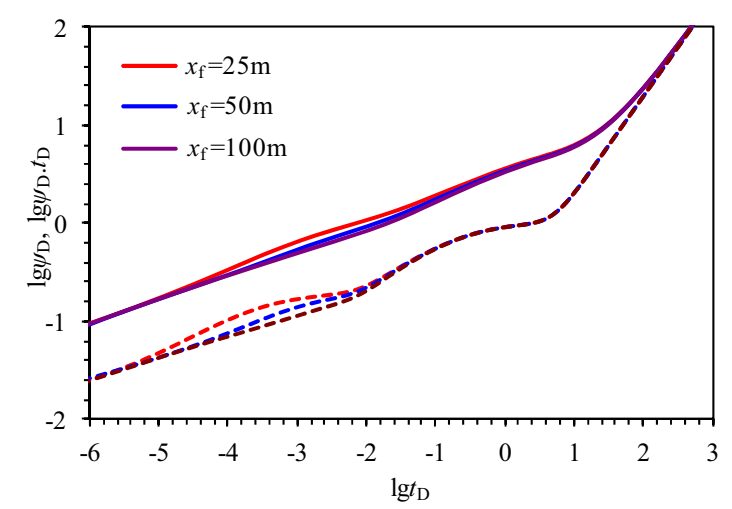

Figure 4. Effect of $x_{\mathrm{f}}$ on the type curves

Fig.5 shows the effect of hydraulic fracture spacing $\left(d_{\mathrm{f}}\right)$ on pseudo-pressure and its derivative curves. With the increase of $d_{\mathrm{f}}$, the early radial flow becomes longer and then the occurrence of elliptical flow regime postpones. In addition, the smaller $d_{\mathrm{f}}$ shows, the larger the corresponding pressure drop becomes, this is mainly because the interference between the hydraulic fractures will be more serious for a smaller $d_{\mathrm{f}}$.

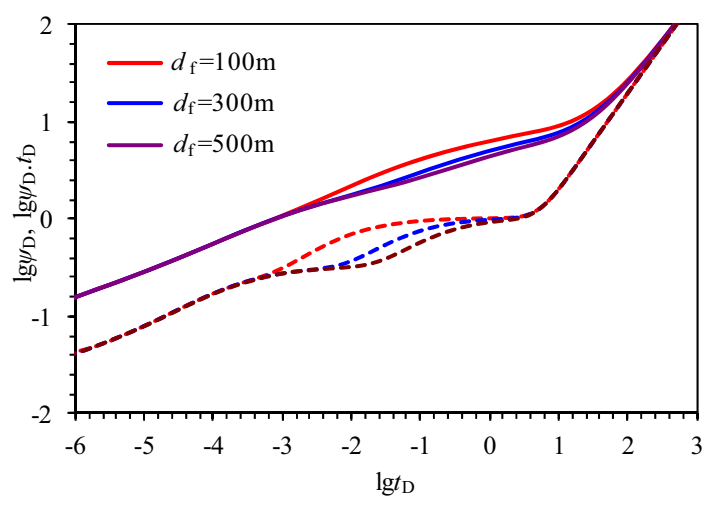

Figure 5. Effect of $d_{\mathrm{f}}$ on the type curves

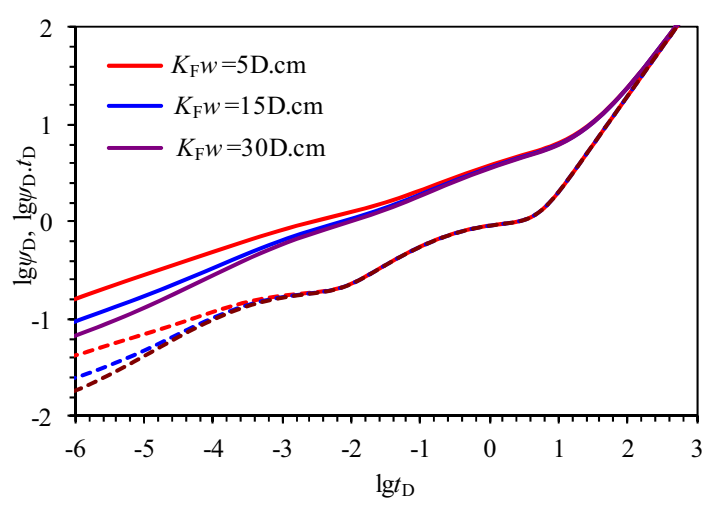

Figure 6. Effect of $K_{\mathrm{Fw}}$ on the type curves

Fig.6 shows the effect of hydraulic fracture conductivity $\left(K_{\mathrm{Fw}}\right)$ on pseudo-pressure and its derivative curves, hydraulic fracture conductivity is the product of hydraulic fracture permeability and its width. It can be seen that the $K_{\mathrm{Fw}}$ mainly affects the early linear flow 
regime, and the bigger $K_{\mathrm{Fw}}$ is, the the smaller the pressure drop will be.

\section{Conclusions}

(1) In this paper, a new pressure transient model of multi-fractured horizontal well based on discrete-fracture model has been built and solved by finite element method.

(2) In this new pressure transient model, five main flow regimes can be observed in transient pressure and its derivative curves.

(3) Hydraulic fracture parameters have great effect on the pressure transient curves. With the decrease of hydraulic fractures, the occurrence time of elliptical flow shows later, even disappears when there is only one hydraulic fracture. The smaller the hydraulic fracture half-length is, the earlier the early radial flow will appear, and the longer the duration will last. The smaller the hydraulic fracture spacing shows, the larger the corresponding pressure drop becomes.

\section{Acknowledgment}

The authors would like to acknowledge the financial support of the Science and Technology Project of China Petroleum and Chemical Industry Association (2014-0102).

\section{References}

1. Gringarten, A. C., Ramey, H. J., 1973. The use of source and green's function in solving unsteady-flow problem in reservoir. SPE Journal, 285-296, SPE 3818 .

2. Ozkan, E., 1988: "Performance of horizontal wells," Ph.D. Dissertation, The University of Tulsa, May (1988).

3. Raghavan, R., Chin-Cheng Chen, Bijin Agarwal, 1997. An analysis of horizontal wells intercepted by multiple fractures. SPE Journal, SPE-27652.

4. Zerzar, A., Bettam, Y., Tiab, D., 2003. Interpretation of multiple hydraulically fractured horizontal wells in closed systems. In: SPE 84888 presented at SPE International Improved Oil Recovery Conference in Asia Pacific, Kuala Lumpur, Malaysia.

5. Al-Kobaisi M., Ozkan E., 2004. A hybrid numerical analytical model of finite conductivity vertical fractures intercepted by a horizontal well. In: SPE 92040 presented at SPE International Petroleum Conference in Puebla, Mexico.

6. Valko, P.P. and Amini, S., 2007. The method of distributed volumetric sources for calculating the transient and pseudosteady-state productivity of complex well-fracture configuration. In: SPE Paper 106279 Presented at the SPE Hydraulic Fracturing Technology Conference, College Station, TX.

7. Zhao, Y. L., Zhang, L. H., Zhao, J. Z, et al., 2013. "Triple porosity"modeling of transient well test rate decline analysis for multi-fractured horizontal well in shale gas reservoirs. J. Petrol. Sci. Eng. 110, $253 \mathrm{e} 262$.

8. Yao, J., Liu, P. Y., Wu, M. L., Well test analysis of fractured horizontal well in fractured reservoir. Journal of China University of Petroleum, 2013, 37(5):107-113+119.

9. Ozkan, E., Brown, M., Raghavan, R., et al., 2009. Comparison of fractured horizontal well performance in conventional and unconventional reservoirs. In: SPE Paper 121290-MS Presented at SPE Western Regional Meeting, San Jose, California, USA.

10. Ozkan, E., Brown, M., Raghavan, R., et al., 2011. Comparison of fractured-horizontal well performance in tight sand and shale reservoirs. SPE Reser. Eval. Eng. 14 (2), 248-259.

11. Stalgorova, E., Mattar, L., 2012a. Practical Analytical model to simulate production of horizontal wells with branch fractures. In: SPE Paper 162515 Presented at SPE Canadian Unconventional Resource Conference, Calgary, Alberta, Canada.

12. Stalgorova, E., Mattar, L., 2012b. Analytical model for history matching and forecasting production in multifrac composite systems. In: SPE Paper 162516MS Presented at SPE Canadian Unconventional Resource Conference, Calgary, Alberta, Canada.

13. Samandarli, O., McDonald, B., Barzola, G., et al., 2014. Understanding shale performance: performance analysis workflow with analytical models in eagle ford shale play. In: SPE Paper 169004-MS Presented at the SPE Unconventional Resources Conference, Woodlands, Texas, USA.

14. Clarkson, C. R. 2013. Production data analysis of unconventional gas wells: workflow. Int. J. Coal Geol. 109-110 (1 April): 147-157.

15. Clarkson, C. R., Williams-Kovacs, J. D., Qanbari, F., et al., 2014. History matching and forecasting tight/shale gas condensate wells using combined analytical, semi- analytical, and empirical methods. In: SPE Paper 171593-MS Presented at the SPE/CSUR Unconventional Resources ConferenceCanada, Calgary, Alberta, Canada.

16. Sureshjani M. H., Clarkson, C. R.. An analytical model for analyzing and forecasting production from multifractured horizontal wells with complex branched-fracture geometry. SPE Reservoir Evaluation \& Engineering, 2015, 18(3). SPE176025-PA.

17. Karimi-Fard M. and Firoozabadi A., Numerical simulation of water injection in fractured media using the discrete-fracture model and the galerkin method. SPE Reservoir Evaluation \& Engineering, 2003, 6(2), 117-126. SPE-83633-PA. 